\title{
Adrenocortical Adenoma and Carcinoma: Histopathological and Molecular Comparative Analysis
}

Alexander Stojadinovic, M.D., Murray F. Brennan, M.D., Axel Hoos, M.D., Ph.D., Atilla Omeroglu, M.D., Denis H.Y. Leung, Ph.D., Maria E. Dudas, Aviram Nissan, M.D., Carlos Cordon-Cardo, M.D., Ronald A. Ghossein, M.D.

Departments of Surgery (MFB, AH, AN) and Pathology (AH, AO, MeD, CC-C, RAG), Memorial SloanKettering Cancer Center, New York, New York; Department of Surgery (AS), Walter Reed Army Medical Center, Washington, D.C.; and School of Economics and Social Sciences (DHYL), Singapore Management University, Singapore

We compared histomorphological features and molecular expression profiles of adrenocortical adenomas (ACAd) and carcinomas (ACCa). A critical histopathological review (mean, 11 slides per patient) was conducted of 37 ACAd and 67 ACCa. Paraffinembedded tissue cores of ACAd $(n=33)$ and ACCa $(n=38)$ were arrayed in triplicate on tissue microarrays. Expression profiles of p53, mdm-2, p21, Bcl-2, cyclin D1, p27, and Ki-67 were investigated by immunohistochemistry and correlated with histopathology and patient outcome using standard statistical methodology. Median follow-up period was 5 years. Tumor necrosis, atypical mitoses, and $>1$ mitosis per 50 high-power fields were factors that were highly specific for ACCa $(P<.001)$. Number $(0$ to 4) of unfavorable markers [Ki-67 (+), p21 (+), p27 (+), mdm-2(-)] expressed was significantly associated with mitotic activity and morphologic index (i.e., number of adverse morphologic features) and highly predictive of malignancy $(P<.001)$. Ki-67 overexpression occurred in 0 ACAd and $36 \%$ ACCa $(P<.001)$ and was significantly associated with mitotic rate and unfavorable morphologic in$\operatorname{dex}(P<.001)$. Tumor necrosis, atypical mitoses, $>5$ mitoses per 50 high-power fields, sinusoidal invasion, histologic index of $>5$, and presence of more than two unfavorable molecular markers were associated significantly with metastasis in ACCa. Wellestablished histopathologic criteria and Ki-67 can specifically distinguish ACCAd from ACCa. Tumor cell proliferation (Ki-67) correlates with mitotic activity and morphologic index. Tumor morphology is

Copyright () 2003 by The United States and Canadian Academy of Pathology, Inc.

VOL. 16, NO. 8, P. 742, 2003 Printed in the U.S.A.

Date of acceptance: May 1, 2003.

Address reprint requests to: Alexander Stojadinovic, M.D., Walter Reed Army Medical Center, General Surgery Service, 6900 Georgia Avenue, N.W., Wash ington, D.C. 20307; fax: 202-782-1234; e-mail: ta.stojadinovic@verizon.net.

DOI: 10.1097/01.MP.0000081730.72305.81 a better predictor of metastatic risk in ACCa than current immunohistochemistry-detected cell cycle regulatory and proliferation-associated proteins.

KEY WORDS: Adenoma, Adrenal, Carcinoma, IHC, Tissue microarray.

Mod Pathol 2003;16(8):742-751

Adrenocortical carcinoma is a highly aggressive, rare endocrine malignancy. However, benign, clinically occult adrenal adenomas, adrenal "incidentalomas," are encountered frequently by abdominal imaging performed for unrelated indications. The unsuspected adrenal mass is detected in $2-4 \%$ of the general population and in $\leq 6 \%$ of abdominal computed tomography scans in patients aged $60-70$ years $(1,2)$. The size of the adrenal mass is considered to be the most reliable predictor of malignancy. As $8-13.5 \%$ of resected adrenocortical carcinomas are $<5 \mathrm{~cm}$, size alone is an imperfect criterion for malignancy (3).

In a study of metastasizing and nonmetastasizing adrenocortical tumors among 43 patients followed for a median of 11 years, Weiss (4) demonstrated the utility of nine histomorphologic criteria in predicting the biology of adrenocortical neoplasms. The histologic findings most predictive of malignancy were $>5$ mitoses per 50 high-power fields, atypical mitoses, and venous invasion. No single criterion could distinguish benign from malignant tumor biology; all but one of the benign adrenocortical tumors had two or fewer criteria. In a later study, Weiss et al. (5) modified the diagnostic criteria for benign and malignant adrenocortical neoplasms; tumors exhibiting three or more adverse histologic features were considered malignant. A recent study identified six morphologic prognostic factors that correlated significantly with diseasespecific survival in adrenocortical carcinoma: venous, capsular, and adjacent organ invasion; tumor 
necrosis and mitotic rate; and atypical mitosis (6). Both studies identified mitotic activity as the single most important determinant of tumor-related mortality from adrenocortical carcinoma $(5,6)$.

Difficulty remains in differentiating benign from malignant adrenal tumors, particularly in large tumors without invasive features and cellular atypia (7). Nuclear DNA content by flow cytometry correlates poorly with histological and mitotic indices and cannot reliably differentiate between adrenocortical adenoma and carcinoma (8). P53 tumor suppressor gene alterations have been shown to have variable expression and little prognostic value for adrenocortical carcinoma (8-10). Immunohistochemical analyses of antigens that serve as markers of cell proliferation-Ki67, PCNA, and epidermal growth factor receptor-have demonstrated relatively increased expression of these proteins in adrenocortical carcinoma (11-16). No consistent correlations between these cell cycle proteins and histopathology, mitotic index, and outcome could be identified (16).

Multimolecular profiling using immunohistochemical analysis of tissue microarrays has been shown to be an efficient, reproducible, and valid method to study cell cycle regulatory proteins in endocrine neoplasms $(6,17-19)$. In the present study, tissue microarrays and immunohistochemical analysis are applied to a cohort of adrenal tumors. The main objective of the study is to define molecular phenotypes of potential value in distinguishing benign from malignant adrenocortical neoplasms. For that purpose, we compared a group of adrenocortical carcinomas about which we previously published (6) with a group of adrenocortical adenomas from our institution.

\section{MATERIALS AND METHODS}

\section{Patient Search and Inclusion and Exclusion Criteria}

We identified 67 consecutively treated patients with histologically confirmed adrenocortical carcinoma from the Memorial Sloan-Kettering Cancer Center Prospective Endocrine Tumor Database. All patients underwent resection of primary adrenocortical carcinoma and had histopathologic slides available for review. Paraffin-embedded blocks were available for 38 of these patients. We reported on the above-mentioned group of adrenocortical carcinoma patients in a prior publication (6). A search of the Memorial Sloan-Kettering Cancer Center Department of Pathology computerized database was conducted using the terms adrenal and adenoma, identifying 60 patients treated surgically for adrenal adenomas between the years 1990 and 1998. Patients with nonadrenocortical tumors, or those with adrenal hyperplasia or metastasis to the adrenal gland and those having only fine-needle aspiration or biopsy of the adrenal were excluded, leaving 37 patients treated with adrenalectomy with available slides for review. Paraffin blocks were available for 33 cases. All available clinical, pathological, treatment, and follow-up data were reviewed and were updated for 104 patients who underwent adrenalectomy for either adrenocortical adenoma or carcinoma. The role of re-resection and prognostic variables for adrenocortical carcinoma were the subjects of previous reports from our institution $(20,21)$. We later defined the molecular phenotype of normal adrenal tissue and malignant adrenal tumors and compared the morphologic and molecular parameters with one another and correlated these factors with patient outcome in a multivariate analysis (6). The purpose of the present study is to compare the histological features and multimolecular phenotypes of benign and malignant adrenocortical neoplasms.

\section{Treatment}

All patients underwent primary treatment according to standard of care at Memorial SloanKettering Cancer Center with adrenalectomy with or without concomitant solid-organ (pancreas, spleen, kidney, and/or liver) resection as necessary to achieve complete resection of all grossly evident disease. Adjuvant treatment in the form of radiotherapy or chemotherapy was administered as part of standard of care or as part of clinical trials.

\section{Pathologic Review}

All available operative reports and information in the institutional pathology database and pathology reports of the primary tumor were reviewed to confirm completeness of resection. All available autopsy reports were reviewed. Two members (RAG and $\mathrm{AO}$ ) of the pathology department conducted a critical histopathological review of all available slides. A mean of 11 hematoxylin-eosin slides per patient was reviewed in conjunction with the corresponding pathologic record but without knowledge of clinical data. All histologically confirmed adrenocortical adenomas and carcinomas were included in the analysis. All adrenocortical tumors were evaluated according to the following pathologic criteria: gross tumor size and weight, nuclear grade, mitotic rate per 50 high power fields (hpf), tumor cell cytoplasm $(0-25 \%$ and $26-100 \%$ clear $)$ and architecture (diffuse and nondiffuse), presence or absence of atypical mitoses, necrosis, and unequivocal capsular, venous, sinusoidal and adjacent organ invasion $(4,5)$. All adrenocortical carcinomas in this study had three or more of Weiss' 
histologic criteria (4). Mitotic rate was determined by counting 50 high-power fields $(400 \times)$ with an Olympus microscope (U-DO model). The regions of the slide selected were those containing the highest concentrations of mitotic figures. Each 10 highpower fields were counted on a different slide as feasible. Architecture was defined as "diffuse" when sheets of cells without a characteristic pattern of growth comprised $>33 \%$ of the tumor; otherwise, the architecture was categorized as nondiffuse. Endothelial lined vessels with muscle comprising the vessel wall were regarded as a vein, unlike sinusoids, endothelial lined vessels with little supporting tissue. A vein or sinusoid within or external to the adrenal tumor that contained tumor cells within its lumen and was adherent to its wall defined venous or sinusoidal invasion. A tumor completely penetrating the surrounding capsule defined unequivocal capsular invasion.

\section{Clinicopathological Categories}

Clinical data included patient age, gender, presenting symptoms, functional endocrine tumor activity, history of prior malignancy, and radiologic tumor size. During the histopathologic review, microscopic resection margins as well as the 11 previously noted morphologic criteria of Weiss (4) were recorded. Molecular markers studied included Ki67, p53, mdm-2, p21, p27, bcl-2, and cyclin D1.

\section{Definitions}

A primary tumor was defined as a previously untreated, or biopsied (needle aspiration, incisional, or inadequate excisional biopsy), mass before definitive surgical therapy. Patients were considered to have prior cancer if they had a histologically confirmed malignancy other than nonmelanoma skin cancers or carcinoma in situ of the cervix before diagnosis of the adrenal neoplasm. An adrenal neoplasm was considered functional when biochemical analysis confirmed excess adrenocortical hormone secretion. Complete surgical resection was defined as the absence of gross residual disease after surgical excision of the adrenal tumor. Microscopic margins were defined at the time of initial histopathological assessment and confirmed at time of re-review. Microscopic resection margins were categorized as positive (tumor at the inked margin) or negative (no tumor at the inked margin). Follow-up was calculated from the time of primary operation to the date of last follow-up.

\section{Tissue Microarray Construction and} Immunohistochemistry

Paraffin-embedded blocks were available for 33 and 38 patients with adrenocortical adenoma and carcinoma, respectively. Five-micrometer hematoxylin/eosin-stained sections of paraffinembedded sections of benign and malignant adrenocortical tissue were reviewed to confirm diagnosis and target areas of the block for tissue microarray construction, from which 0.6-mmdiameter core biopsies were taken with a precision instrument (Beecher Instruments, Silver Spring, MD) as described elsewhere $(19,22)$. Tissue cores were arrayed in triplicate on a recipient paraffin block (22). Five-micrometer sections of these tissue array blocks were cut and placed on charged polylysine-coated slides. These sections were used for immunohistochemical analysis as described elsewhere $(6,17-19)$. Tissues and cell lines known to express the antigens of interest were used as positive controls. Normal tissues were included on the microarray and were used as baseline controls.

Sections from tissue arrays were deparaffinized, rehydrated in graded alcohols, and processed using the avidin-biotin immunoperoxidase method. The sections underwent microwave oven treatment for 15 minutes in $0.01 \mathrm{M}$-citrate buffer at $\mathrm{pH}$ 6.0. This procedure was followed for all antibodies used in this study, with the exception of Ki-67 antibody that was incubated in preheated $0.05 \%$ trypsin, $0.05 \%$ $\mathrm{CaCl}_{2}$ in $0.05 \mathrm{M}$ Tris- $\mathrm{HCl}(\mathrm{pH}$ 7.6) for 5 minutes at $37^{\circ} \mathrm{C}$ before microwave treatment. After antigen retrieval, slides were incubated in $10 \%$ normal horse serum for 30 minutes, then incubated overnight at $4^{\circ} \mathrm{C}$ in appropriately diluted primary antibody. Mouse anti-human monoclonal antibodies to p53 (Ab-2, clone 1801, 1:500 dilution; Calbiochem, Cambridge, MA), mdm-2 (clone 2A10, 1:500 dilution; provided by Dr. A. Levine, Rockefeller University, New York), p21 (WAF-1 [Ab-1], clone EA10, 1:100 dilution; Calbiochem), p27 (kip-1 [Ab-2], clone DCS72, 1:500 dilution; Oncogene Research Products, Cambridge, MA), cyclin D1 (Ab-3, clone DCS-6, 1:100 dilution; Calbiochem), Ki-67 (clone Mib-1, 1:1000 dilution; DAKO, Glostrup, Denmark), and Bcl-2 (clone 124, 1:154 dilution; DAKO) were used. The anti-p53 antibody detects wild-type and mutated p53. Samples were then incubated with biotinylated anti-mouse immunoglobulins at 1:500 dilution (Vector Laboratories, Inc., Burlingame, CA), followed by avidin-biotin peroxidase complexes (1:25, Vector Laboratories, Inc.) for $30 \mathrm{~min}$ utes. Diaminobenzidine was used as the chromogen, and hematoxylin, as the nuclear counterstain.

Rates of lost cases attributable to tissue damage ranged between 1 and $10 \%$ for the different molecular markers. Immunoreactivity was classified as continuous data (undetectable levels, 0, to homogeneous staining, $100 \%$ ) for all markers. Two investigators (RAG, AO) reviewed and scored slides independently by estimating the percentage of tumor cells showing characteristic staining. The cutoff val- 
ues for tumor cell staining used in the present study were defined based on previously established cutoff values used in prior clinicopathological studies of endocrine neoplasms employing identical reagents $(6,17,18)$. Evaluation of microarray-based data was performed according to a previously established and validated system (19). The cutoff values for tumor cell staining used for all adrenocortical neoplasms in this study were defined as follows: (1) high Ki-67 proliferative index if $>5 \%$ tumor nuclei stained; (2) p53 nuclear overexpression if $>5 \%$ tumor nuclei stained; (3) $\mathrm{mdm}-2$ overexpression if $>50 \%$ tumor nuclei stained; (4) p21 nuclear overexpression if $>10 \%$ of tumor nuclei stained; (5) p27 nuclear overexpression if $>30 \%$ nuclei stained; (6) Bcl-2 overexpression if $>50 \%$ of tumor cells demonstrated cytoplasmic staining; and (7) cyclin D1 overexpression if $>5 \%$ of tumor nuclei stained. Tumors were then grouped into binary categories defined as follows: negative expression (neoplasms below defined cutoff value of immunoreactivity) and positive expression (neoplastic tissues above defined cutoff values of immunoreactivity).

\section{Statistics}

Associations between categorical variables were evaluated using the Fisher's exact test when frequency was small and were carried out using the $\chi^{2}$ test with Yates' correction when frequency was large enough to justify its use. The CochranArmitage test was used to test for trends. Nonparametric comparison of median values across groups was performed for continuous variables using the Wilcoxon/Kruskall-Wallis rank-sum tests. To assess the independent predictive effect of a covariate (tumor size or mitotic rate, for example) for a nominal response (in this case malignancy), a logistic regression model was constructed and parameters were estimated using maximum likelihood. No multivariate analyses were performed for the clinical endpoints because of the moderate number of cases and the fact that only very few cases had not developed the endpoint. The likelihood ratio test was computed for each effect in the model. Confidence limits and odds ratios were calculated for the maximum likelihood parameter estimates. Many of the variables, such as mitotic index and molecular markers, had skewed distributions with a large number of ties at the lower end of their range. For information purposes, the median of the variables is reported where appropriate, but for purpose of analysis, they were analyzed as categorical variables. Statistical analysis was performed using the JMP statistical package (JMP, Cary, NC). A $P$ value of $<.05$ was considered significant.

\section{RESULTS}

\section{Clinical Features}

Median follow-up for those alive at time of last clinic visit was 5.5 years (range, 1.2-16.9 y). The majority $(78.4 \%)$ of benign adrenal tumors were identified among patients with previously treated nonadrenal malignancy during cross-sectional imaging. Adrenalectomy in these patients was performed on the basis of increased suspicion of single-site metachronous distant metastasis. Three $(8.1 \%)$ of these patients were found to have asymptomatic functional tumors during biochemical testing. The fact that functional adrenocortical neoplasms were associated with higher mitotic $(79 \% v s$. $28 \%,>5$ per 50 high-power field, $P<.001)$ and Ki-67 overexpression ( $29 \%$ vs. $8 \%$, positive, $P=.1$ ) than nonfunctional tumors likely reflects the increased proportion of hormonally active malignant to benign lesions ( $45 \%$ vs. $8 \%, P<.001$ ). Median primary tumor size of adrenocortical carcinoma significantly exceeded that of adrenal adenoma (14.5 vs. $2.5 \mathrm{~cm}, P<.001)$. Median size of the adrenal tumors was significantly higher, with high mitotic activity (3.6 vs. $14.9 \mathrm{~cm}: \leq 5$ vs. $>5$ per 50 high-power fields, $P<.001)$ and Ki-67 overexpression (15 vs. $4 \mathrm{~cm}$ : [+] vs. [-] Ki67, $P<.001)$. See Table 1 for a summary of these features.

\section{Morphology}

No benign and $91 \%$ of malignant tumors exceeded $100 \mathrm{~g}$ in weight $(P<.001)$. Although no adenoma in this study had histologic evidence of venous, capsular, sinusoidal, or adjacent organ invasion, these findings were identified in $42 \%, 53 \%$, $94 \%$, and $8 \%$ of adrenocortical carcinomas $(P<$ $.001)$. The majority (87\%) of adrenal adenomas

TABLE 1. Adrenal Adenoma versus Carcinoma, Clinical Features

\begin{tabular}{|c|c|c|c|}
\hline Characteristic & $\begin{array}{c}\text { Adenoma } \\
(n=37) \\
n(\%)\end{array}$ & $\begin{array}{c}\text { ACC } \\
(n=67) \\
n(\%)\end{array}$ & $P$ \\
\hline Gender & & & 0.721 \\
\hline Female & $23(62.2 \%)$ & $44(65.7 \%)$ & \\
\hline Male & $14(37.8 \%)$ & $23(34.3 \%)$ & \\
\hline Median age, years (range) & $62(41-84)$ & $47(2-77)$ & 0.001 \\
\hline Symptoms & & & $<0.001$ \\
\hline Asymptomatic & $31(83.8 \%)$ & $2(2.9 \%)$ & \\
\hline Endocrine & $3(8.1 \%)$ & $30(44.8 \%)$ & \\
\hline Pain & $0(0.0 \%)$ & $14(20.9 \%)$ & \\
\hline Palpable mass & $0(0.0 \%)$ & $3(4.5 \%)$ & \\
\hline Other & $0(0.0 \%)$ & $5(7.5 \%)$ & \\
\hline Unknown & $3(8.1 \%)$ & $13(19.4 \%)$ & \\
\hline Tumor status & & & $<0.001$ \\
\hline Secretory & $3(8.1 \%)$ & $30(44.8 \%)$ & \\
\hline Non-secretory & $31(83.8 \%)$ & $24(35.8 \%)$ & \\
\hline Unknown & $3(8.1 \%)$ & $13(19.4 \%)$ & \\
\hline Coexisting malignancy & $29(78.4 \%)$ & N/A & $\mathrm{N} / \mathrm{A}$ \\
\hline \multirow[t]{2}{*}{ Median tumor size, cm (range) } & & & $<0.001$ \\
\hline & $2.5(0.9-7.0)$ & $14.5(4.0-28.5)$ & \\
\hline
\end{tabular}


demonstrated nuclear features consistent with $\mathrm{Fu}$ hrman nuclear Grade 1 or 2; these findings were evident in only one $(1.5 \%)$ adrenal carcinoma $(P<$ $.001)$. Tumor necrosis and atypical mitotic figures were seen exclusively in adrenocortical carcinomas.

Mitotic figures were observed in all but two (3.0\%) primary adrenal malignancies. A single mitotic figure was identified among 1850 high-power fields examined in adrenocortical adenomas. Although not specific for malignancy, mitotic figures were an infrequent finding among benign adrenal tumors ( $3 \%$ vs. $97 \%, P<.001)$. Mitosis of $>1$ per 50 high-power fields was specific for adrenocortical carcinoma in this patient cohort.

Most (82\%) malignant adrenal tumors had diffuse architecture consisting of sheets of cells without a characteristic pattern of growth in $>33 \%$ of the tumor; however, this feature was not observed in any benign adrenal tumor $(P<.001)$. Zero to $25 \%$ clear tumor cell cytoplasm was not a specific manifestation of malignancy, as $13.5 \%$ of adenomas demonstrated this finding.

The frequency of 12 morphologic features among benign and malignant adrenocortical tumors is shown in Table 2. These adverse pathologic features include tumor weight of $>250 \mathrm{~g}$; nuclear Grade III or IV; necrosis; diffuse architecture; 0-25\% clear cytoplasm; presence of capsular, venous, sinusoidal or adjacent organ invasion; mitotic activity of $>5$ per 50 high-power fields; and mitotic atypia. The number of adverse morphologic features (morphologic index) was used in the analysis.

\section{Immunohistochemical Profiling of Cell Cycle Regulatory Proteins}

See Table 3 for a summary of these profiles. The patterns of p53 expression and those of important related molecules, mdm-2, p21, and Bcl-2 were investigated. Expression patterns of two regulators of cell cycle progression, cyclin D1 and p27, as well as the cell proliferation marker, Ki-67, were evaluated as well. The anti-p53 antibody detects wild-type and mutated p53. The half-life and expression of p53 protein is low and therefore undetectable by immunohistochemistry. In malignant cells, most p53 mutations lead to products that are not ubiquitinated, accumulate in the nuclei, and can be demonstrated by immunohistochemistry. Immunohistochemical profiling of p53 expression demonstrated absence of nuclear staining in all but two $(5.4 \%)$ adrenocortical carcinomas.

Although the majority of adrenal neoplasms demonstrated nuclear staining for the inactivating p53-binding protein, mdm-2, nuclear overexpression of this molecule was infrequent ( $28 \%$ overall). The overexpression of mdm-2 among adrenocortical adenomas and carcinomas was not significantly
TABLE 2. Adrenal Adenoma versus Carcinoma, Morphologic Features

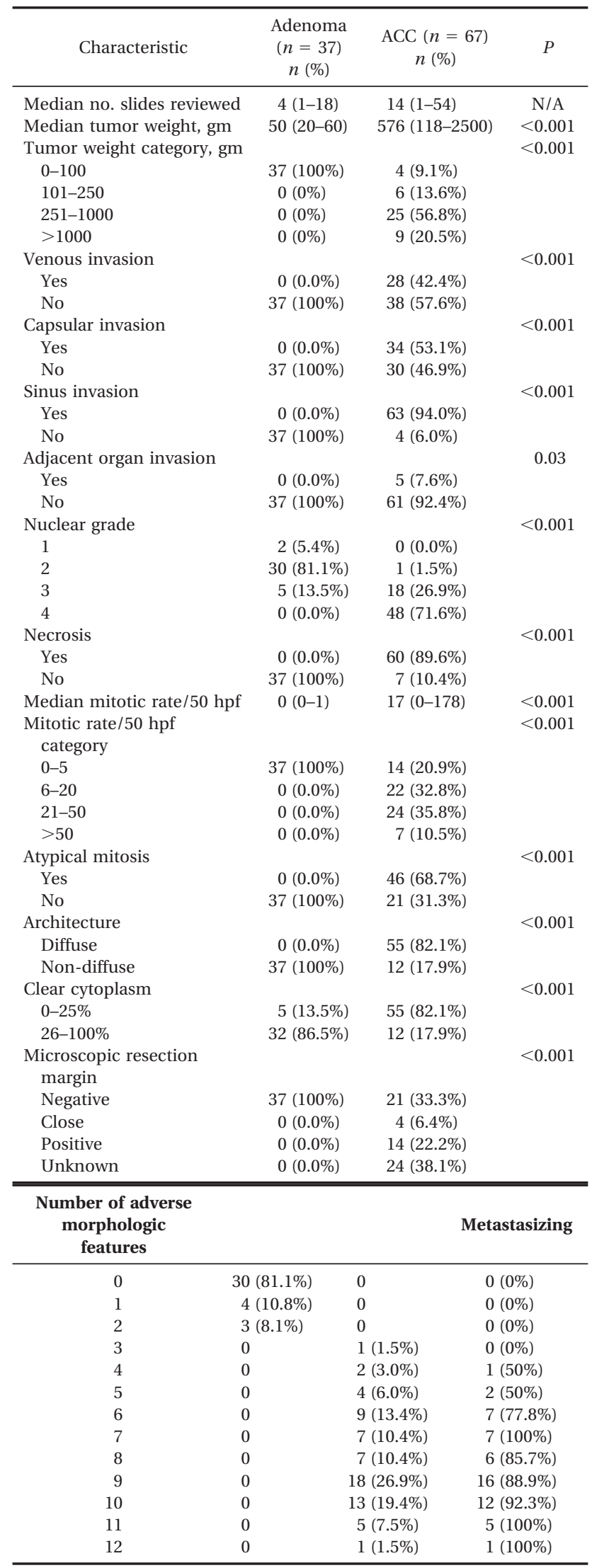

Numbers $<67$ indicate only those variables that could be determined with certainty after review of the histopathology slides and pathology reports. 
TABLE 3. Cell Cycle Regulator Expression in Adrenocortical Adenoma versus Carcinoma

\begin{tabular}{lccc}
\hline Molecular Marker & $\begin{array}{c}\text { Adenoma } \\
(n=33)\end{array}$ & $\begin{array}{c}\text { ACC } \\
(n=38)\end{array}$ & $P$ \\
(positive percent cutoff) & $n(\%)$ & $n(\%)$ & $<0.001$ \\
\hline Ki-67 (5\%) & $33(100 \%)$ & $20(64.5 \%)$ & \\
Negative & $0(0 \%)$ & $11(35.5 \%)$ & \multirow{2}{*}{0.107} \\
Positive & $38(100 \%)$ & $35(94.6 \%)$ & \\
p53 (5\%) & $0(0 \%)$ & $2(5.4 \%)$ & 0.131 \\
Negative & $21(63.6 \%)$ & $28(80 \%)$ & \\
Positive & $12(36.4 \%)$ & $7(20 \%)$ & 0.006 \\
mdm-2 (50\%) & & & \\
Negative & $21(63.6 \%)$ & $11(30.6 \%)$ & \\
Positive & $12(36.4 \%)$ & $25(69.4 \%)$ & \\
p21 (10\%) & & & \\
Negative & $10(31.3 \%)$ & $2(5.6 \%)$ & \\
Positive & $22(68.8 \%)$ & $34(94.4 \%)$ & N/A \\
p27 (30\%) & & & \\
Negative & $33(100 \%)$ & $38(100 \%)$ & \\
Positive & $0(0 \%)$ & $0(0 \%)$ & \\
Bcl-2 (10\%) & $33(100 \%)$ & $38(100 \%)$ & \\
Negative & $0(0 \%)$ & $0(0 \%)$ & \\
Positive & & & \\
Cyclin D1 (5\%) & & & \\
Negative & & & \\
Positive & & & \\
\hline
\end{tabular}

Number of patients in subgroup $<$ total number in respective group reflects tissue loss during specimen micro array processing.

different (36\% vs. 20\%, $P=.13$ ). Nuclear p21 expression was present in both benign and malignant adrenocortical tumors, although a significantly lower proportion of adenomas manifested the p21positive phenotype ( $36 \%$ vs. $69 \%, P=.006)$.

The lowest rates of expression among the investigated markers were observed for the antiapoptotic molecule, Bcl-2, and the regulator of the G1 checkpoint of the cell cycle, cyclin D1. Low to absent nuclear immunostaining for these two molecules was identified in all benign and malignant adrenal tumors. The highest rate of expression for both benign and malignant adrenocortical tumors was for the cyclin-dependent kinase inhibitor, p27 protein; however, a significantly lower proportion of adenomas manifested the p27-positive phenotype (69\% vs. 94\%, $P=.009$ ).

Tumor cell proliferative activity was evaluated by immunostaining against the Ki-67 antigen. No patient with adrenocortical adenoma in this study demonstrated a Ki-67-positive phenotype, defined as $>5 \%$ of tumor cells with nuclear immunoreactivity. Ki-67 overexpression was present in 35\% of adrenocortical carcinomas (Fig. 1). Thus, differential molecular expression distinguished between the benign and malignant adrenal tissue, as there was statistically significant differential expression of the following cell cycle regulatory proteins: Ki-67, p21, and p27 (Table 3).

As no cases in this study demonstrated overexpression of Bcl-2 or cyclin D1, and only two cases overexpressed p53, these markers have little value in predicting adrenocortical tumor biology. Given the moderate number of cases in the tissue mi- croarray, we used the number of markers with unfavorable expression pattern for analytic purposes. Overexpression of Ki-67, p21, and p27 were predictive of malignancy, and overexpression of $\mathrm{mdm}-2$ correlated with benign adrenal tumors by univariate analysis. Therefore, Ki-67 (+), p21 (+), p27 (+), or mdm-2 (-) was regarded as an unfavorable molecular expression pattern (Fig. 1). Each tumor could have zero to four unfavorable molecular markers. The number of unfavorable markers expressed was highly predictive of malignancy, as the proportions of carcinomas expressing zero, one, two, three, and four unfavorable molecular markers were $0,12 \%, 44 \%, 72 \%$, and $100 \%$, respectively (Table $4, P<.001)$.

\section{Morphologic and Molecular Correlations}

A progressive increase in Ki-67 proliferative index was observed with increasing mitotic rate (Table 5 , $P<.001)$. No similar significant association was evident between mitotic rate and either p21 $(P>$ $0.7)$ or p27 $(P>0.1)$. Ki-67 expression was significantly associated with morphologic index, as Ki-67 overexpression was significantly greater for tumors manifesting $>5$ than $\leq 5$ of 12 adverse morphologic features (Table 5, $P<.001$ ). Significant associations also existed between the p27 expression and pathologic features (Table 5, $P<.05$ ), but no significant association was found between p21 expression and morphologic index $(P=.08)$. There were significant associations between both mitotic rate $(P<.001)$ and morphologic index $(P<.001)$ with number of unfavorable molecular markers expressed (Table 6).

\section{Morphologic and Molecular Predictors of Metastatic Adrenocortical Carcinoma}

At the time of last follow-up, 47 patients had died of disease, 13 had died of other causes, 8 were alive with distant metastases, 31 had no evidence of disease, and 5 were lost to follow-up. The majority $(57 / 67,85 \%)$ of patients with adrenocortical carcinoma developed distant metastasis. The number of adverse morphologic features was associated with risk of distant metastasis, as $43 \%$ of tumors demonstrating five or fewer and $90 \%$ of those with more than five pathologic features developed systemic spread of disease $(P<.01)$. Histological factors that were significantly associated with distant metastasis of adrenocortical carcinoma included the presence of tumor necrosis $(P<.01),>5$ mitosis $/ 50 \mathrm{hpf}$ $(P<.01)$, atypical mitoses $(P=.05)$, and sinusoidal invasion $(P<.01)$. No statistically significant correlation could be identified between distant metastasis and any individual investigated molecular markers. The number of unfavorable markers expressed was highly predictive of metastatic spread, 
Adenoma

Ki 67-

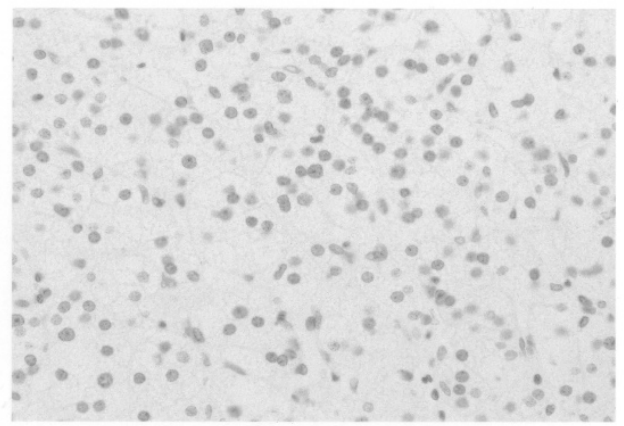

p27-

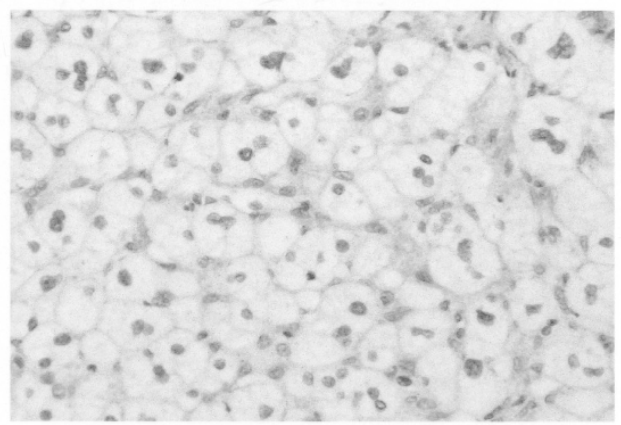

p21-

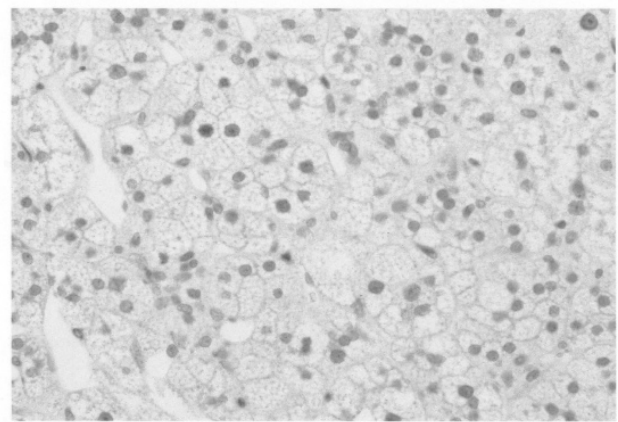

Carcinoma
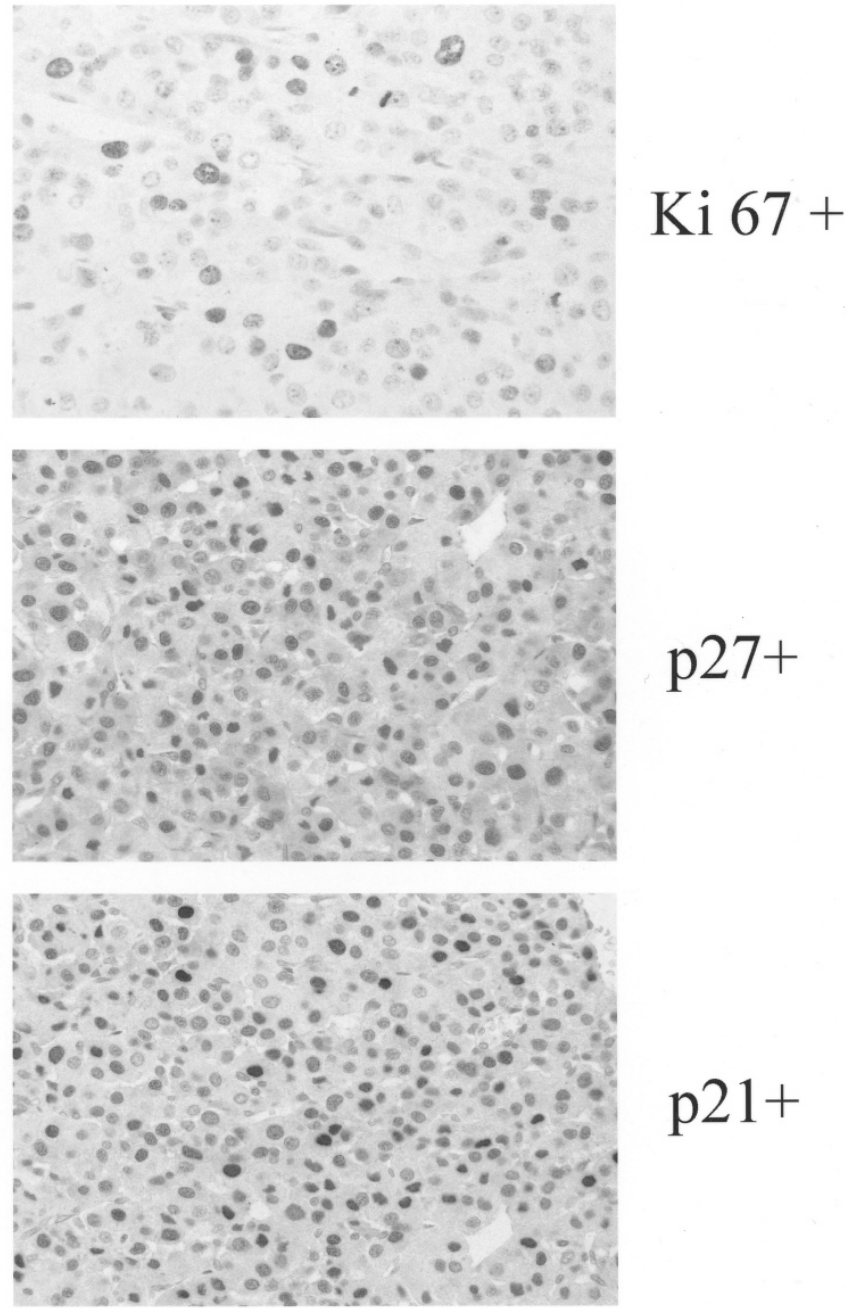

p21+

FIGURE 1. Representative immunophenotypes according to type of adrenocortical tumor based on cell cycle regulatory proteins Ki 67 , p27, and p21. Immunopositivity is defined as nuclear staining for these three markers. Original magnification, $200 \times$.

TABLE 4. Association of Number of Unfavorable Molecular Markers Expressed [Ki-67 (+), p21 (+), p27 (+), mdm2 (-)] with Adrenocortical Tumor Type

\begin{tabular}{lrrrrr}
\hline \multirow{2}{*}{ Tumor Type } & \multicolumn{5}{c}{ Number of Unfavorable Markers } \\
\cline { 2 - 6 } & 0 & \multicolumn{1}{c}{1} & 2 & 3 & 4 \\
\hline Adenoma & 2 & 14 & 9 & 7 & 0 \\
Carcinoma & 0 & 2 & 7 & 18 & 4 \\
Total & 2 & 16 & 16 & 25 & 4 \\
\hline
\end{tabular}

as the proportions of carcinomas with distant metastases expressing zero, one, two, three, and four unfavorable molecular markers were $0,13 \%, 38 \%$, $48 \%$, and $75 \%$, respectively.

\section{DISCUSSION}

The development of advanced cross-sectional imaging has led to earlier diagnosis of malignant adrenocortical tumors, thereby increasing the challenge of differentiating benign from malignant pathology on the basis of histomorphologic features alone $(3,23)$. Most adrenocortical adenomas can be distinguished from carcinomas by permanent section microscopy based on well-defined, uniformly agreed-upon pathologic criteria (4-6). However, the specific histologic diagnosis of adrenal neoplasms can be challenging. Controversy exists over the usefulness of immunohistochemical analysis of biomarkers in indeterminate cases. The purpose of this study was to determine whether immunohistochemical molecular profiling based on tissue microarrays may identify markers useful in distinguishing benign from malignant adrenocortical neoplasms. The pathologic criteria of Weiss et al. (4) were applied in the present histopathological analysis of adrenocortical neoplasms, and carcinomas were defined as tumors demonstrating three or more histologic criteria. No adenoma studied demonstrated venous, capsular, sinusoidal, or adjacent organ invasion; tumor necrosis; or atypical mi- 
TABLE 5. Association of Ki-67, p21, and p27 Expression with Mitotic Rate and Morphologic Index

\begin{tabular}{|c|c|c|c|c|c|c|}
\hline \multirow{2}{*}{ Marker } & \multicolumn{2}{|c|}{ Morphologic Index } & \multicolumn{4}{|c|}{ Mitotic Rate/50 hpf } \\
\hline & $\leq 5$ & $>5$ & $0-5$ & $6-20$ & $21-50$ & $>50$ \\
\hline Ki-67 (-) & $37(71 \%)$ & $15(29 \%)$ & $41(79 \%)$ & $7(8 \%)$ & $4(13 \%)$ & $0(0 \%)$ \\
\hline $\mathrm{Ki}-67(+)$ & $0(0 \%)$ & $11(100 \%)$ & $0(0 \%)$ & $3(55 \%)$ & $6(27 \%)$ & $2(18 \%)$ \\
\hline p21 (-) & $21(66 \%)$ & $11(34 \%)$ & $22(69 \%)$ & $4(12 \%)$ & $5(16 \%)$ & $1(3 \%)$ \\
\hline p21 (+) & $16(44 \%)$ & $20(56 \%)$ & $20(56 \%)$ & 7 (19\%) & $8(22 \%)$ & $1(3 \%)$ \\
\hline p27 (-) & $10(83 \%)$ & $2(17 \%)$ & $11(92 \%)$ & $1(8 \%)$ & $0(0 \%)$ & $0(0 \%)$ \\
\hline p27 (+) & $26(47 \%)$ & $29(53 \%)$ & $30(54 \%)$ & $10(18 \%)$ & $13(24 \%)$ & $2(4 \%)$ \\
\hline
\end{tabular}

TABLE 6. Association of Number of Unfavorable Molecular Markers Expressed [Ki-67 (+), p21 (+), p27 (+), mdm2 (-)] with Mitotic Rate and Morphologic Index

\begin{tabular}{|c|c|c|c|c|c|c|}
\hline \multirow{2}{*}{ No. Factors } & \multicolumn{2}{|c|}{ Morphologic Index } & \multicolumn{4}{|c|}{ Mitotic Rate/50 hpf } \\
\hline & $\leq 5$ & $>5$ & $0-5$ & $6-20$ & $21-50$ & $>50$ \\
\hline 0 & 2 & 0 & 2 & 0 & 0 & 0 \\
\hline 1 & $14(88 \%)$ & $2(12 \%)$ & $15(94 \%)$ & $1(6 \%)$ & 0 & 0 \\
\hline 2 & $9(56 \%)$ & $7(44 \%)$ & $9(56 \%)$ & $3(19 \%)$ & $4(25 \%)$ & 0 \\
\hline 3 & $11(44 \%)$ & $14(56 \%)$ & $14(56 \%)$ & $4(16 \%)$ & $6(24 \%)$ & $1(4 \%)$ \\
\hline 4 & $0(0 \%)$ & $4(100 \%)$ & 0 & 1 & 2 & 1 \\
\hline
\end{tabular}

totic figures. Mitotic rate $>1$ per 50 high-power fields was specific for adrenocortical carcinoma. Identification of the morphologic characteristics requires pathologic experience with endocrine neoplasms and meticulous examination of $\geq 50$ high-power fields. Variable interobserver results may occur in the quantification of mitotic activity, the identification of atypical mitoses, and the assessment of capsular and vascular invasion. We have previously validated the currently applied method of tissue microarrays for high-throughput immunohistochemical analysis of proteins involved in cell cycle regulation, cellular proliferation, and apoptosis $(18,19,24)$. Investigation of cell-cyclerelated molecules with this technique has been employed to study a variety of endocrine neoplasms (6, 17-18).

The most frequently detected mutations in human malignancy involve the p53 tumor suppressor gene $(25,26)$. Point mutations and/or deletions inactivate p53, allowing unchecked progression of cells containing damaged DNA through the S phase of the cell cycle, thereby supporting the development of a neoplastic phenotype $(9,10,25,26)$. This and prior studies have demonstrated low to absent p53 immunoreactivity in adrenal adenomas; however, the rate of immunopositivity among carcinomas has been highly variable, ranging from $5 \%$ in this study to $52 \%(6,9,10,15)$. This lack of reproducibility among investigators makes it unlikely that p53 alone is of practical use in differentiating benign from malignant adrenocortical tumors. This may be related to antibody selection, inability of the antibody to recognize a protein with altered configuration, or antigen retrieval methodology.

Murine double minute (mdm-2) binds p53 and induces its degradation and appears to be a com- mon mechanism of p53 inactivation in human malignancy (27). Overexpression of $\mathrm{mdm}-2$ was associated with benign tumor biology in this study and with normal adrenal histology in a prior study (6). There were no significant differences in the mdm-2 positivity rate between adrenocortical adenomas and carcinomas that precluded its use in the differential diagnosis. Wild-type p53 protein and p53independent cellular growth-promoting factors induce overexpression of the cyclin-dependent kinase inhibitor, p21, that leads to cell cycle arrest. No relational pattern could be identified for p21 and mdm-2 overexpression in the same adrenal tumors. However, this and a previous study found significant differential expression of p21 among normal, benign, and malignant adrenal tumors (6). The absence of p21 overexpression has been associated with adverse outcome in other solid-organ tumors (28-30). The present data suggest that p21 is not sufficiently specific for the diagnosis of adrenal malignancy and that nor is it predictive of metastatic risk.

In adenomas as well as in carcinomas, there was no overexpression of the anti-apoptotic molecule, Bcl-2, and the regulator of the G1-S transition of the cell cycle, cyclin D1. Cyclin D1 expression has been found to be uniformly low among normal and neoplastic thyroid, parathyroid, and adrenal tissues; Bcl-2 is overexpressed in thyroid and parathyroid, unlike the case in adrenal tumors, underscoring the marked variability of molecular expression among endocrine neoplasms $(6,17,18)$.

Up-regulation of the cyclin-dependent kinase inhibitor, p27, has been shown to result in cell-cycle arrest and apoptosis $(31,32)$. Although a significantly lower proportion of adenomas than carcinomas manifested the p27-positive phenotype in this 
study, p27 immunostaining lacks the specificity to be of any value in distinguishing between malignant and benign adrenocortical tumors.

We evaluated adrenocortical tumor cell proliferation by nuclear Ki-67 immunostaining. No adrenocortical adenoma in this study demonstrated a Ki-67-positive phenotype. High Ki-67 expression was more frequently identified in adrenocortical carcinoma than adenoma, supporting findings of earlier studies and validating the value of this molecular marker in differentiating benign from malignant adrenocortical tumors $(12,13,16)$. The association between Ki-67 expression and mitotic activity has been reported earlier by Nakazumi et al. (16) and Goldblum et al. (12). The present study supports and extends these findings to a large, pathologically well-characterized cohort with adrenocortical neoplasms. The number of adverse morphologic features displayed by the adrenocortical tumor correlated significantly with expression of Ki-67. This association has not been reported previously, possibly because of the relatively small sample size of patients with adrenocortical carcinoma in earlier studies (12-16). The morphologic analogues of tumor cell proliferation as measured by Ki-67 immunostaining appear to be mitotic activity and the number of adverse pathologic features expressed by the adrenocortical tumor. Because Ki-67 interpretation is less subjective than mitotic count, it can be used by less experienced morphologists and can lead to better interobserver agreement in the diagnosis of malignancy in adrenocortical tumors.

We identified Ki-67, p21, and p27 overexpression and mdm-2 underexpression to be associated with adrenal malignancy. We found the criterion of number ( 0 to 4$)$ of unfavorable markers expressed [Ki-67(+), p21(+), p27 (+), mdm-2(-)] useful for predicting malignancy. The phenotype Ki-67(+) $p 21(+) p 27(+) m d m-2(-)$ was only seen in malignant cases in this study. However, only $13 \%$ of carcinomas expressed this phenotype. Among nine cases with the Ki-67(-) p21(-) p27 (-) mdm-2(+) phenotype, only one was malignant. Albeit few in number, all p53(+) tumors were malignant. Although specific, immunohistochemically defined, multimolecular phenotypes can support the diagnosis of adrenocortical adenoma, they cannot be relied upon for definitive diagnosis of adrenocortical carcinoma in the absence of supportive morphologic criteria, because these molecular markers are not sensitive enough even when combined.

Weiss et al. (5) defined specific morphologic characteristics of adrenocortical carcinoma and found that only mitotic activity correlated significantly with patient survival. The majority of tumorrelated deaths associated with adrenocortical carcinoma occur as a result of distant spread of disease. In this study, we correlated histopathological and molecular parameters with risk of metastasis in adrenocortical carcinomas. Although individual marker expression did not provide insights into the metastatic potential of adrenocortical carcinoma, the number of unfavorable markers expressed $[\mathrm{Ki}-67(+), \mathrm{p} 21(+), \mathrm{p} 27(+), \mathrm{mdm}-2(-)]$ was highly predictive of distant spread of disease. Tumor morphology was the dominant determinant of distant disease progression. Histological assessment remains the cornerstone of the diagnosis and assessment of biology for adrenocortical neoplasms at the present time.

Acknowledgments: The authors gratefully acknowledge the important contributions of Robin Howard, Biostatistics Section, Department of Clinical Investigation, Walter Reed Army Medical Center in the statistical analysis of this data.

\section{REFERENCES}

1. Reincke M, Beuschlein F, Slawik M, Borm K. Molecular adrenocortical tumorigenesis. Eur J Clin Invest 2000;30:63-8.

2. Mantero F, Arnaldi G. Management approaches to adrenal incidentalomas: a view from Ancona, Italy. Endocrinol Metab Clin North Am 2000;29:107-25.

3. Barnett CC, Varma DG, El-Naggar AK, Dackiw AP, Porter GA, Pearson AS, et al. Limitations of size as a criterion in the evaluation of adrenal tumors. Surgery 2000;128:973-83.

4. Weiss LM. Comparative histologic study of 43 metastasizing and nonmetastasizing adrenocortical tumors. Am J Surg Pathol 1984;8:163-9.

5. Weiss LM, Medeiros LJ, Vickery AL. Pathologic features of prognostic significance in adrenocortical carcinoma. Am J Surg Pathol 1989;13:202-6.

6. Stojadinovic A, Ghossein RA, Hoos A, Nissan A, Brennan MF, Jaques DP. Adrenocortical carcinoma: clinical, morphologic and molecular characterization. J Clin Oncol 2002;20:941-50.

7. Grondal S, Cedermark B, Eriksson B, Grimelius L, Harach R, Kristoffersson A. Adrenocortical carcinoma: a retrospective study of a rare tumor with a poor prognosis. Eur J Surg Oncol 1990;16:500-6.

8. Haak HR, Cornelisse CJ, Hermans J, Cobben L, Fleuren GJ. Nuclear DNA content and morphological characteristics in the prognosis of adrenocortical carcinoma. Br J Cancer 1993;68:151-5.

9. Reincke M, Karl M, Travis WH, Mastorakos G, Allolio B, Linehan HM. p53 mutations in human adrenocortical neoplasms: immunohistochemical and molecular studies. J Clin Endocrinol Metab 1994;78:790-4.

10. McNicol AM, Nolan CE, Struthers AJ, Farquharson MA, Herman J, Haak HR. Expression of p53 in adrenocortical tumours: clinicopathological correlations. J Pathol 1997;181: $146-52$.

11. Suzuki T, Sasano H, Nisikawa T, Rhame J, Wilkinson DS, Nagura H. Discerning malignancy in human adrenocortical neoplasms: utility of DNA flow cytometry and immunohistochemistry. Mod Pathol 1992;5:224-30.

12. Goldblum JR, Shannon R, Kaldjian EP, Thiny M, Davenport $\mathrm{R}$, Thompson $\mathrm{N}$, et al. Immunohistochemical assessment of proliferative activity in adrenocortical neoplasms. Mod Pathol 1993;6:663-8. 
13. Sasano H, Imatani A, Shizawa S, Suzuki T, Nagura H. Cell proliferation and apoptosis in normal and pathologic human adrenal. Mod Pathol 1995;8(1):11-7.

14. Iino K, Sasano H, Yabuki N, Oki Y, Kikuchi A, Yoshimi T, et al. DNA topoisomerase $11 \alpha$ and Ki-67 in human adrenocortical neoplasms: a possible marker of differentiation between adenomas and carcinomas. Mod Pathol 1997;10:901-7.

15. Edgren M, Eriksson B, Wilander E, Westlin JE, Nilsson S, Oberg K. Biological characteristics of adrenocortical carcinoma: a study of p53, IGF, EFR-r, Ki-67 and PCBA in 17 adrenocortical carcinomas. Anticancer Res 1997;17:1303-10.

16. Nakazumi H, Sasano H, Iino K, Ohashi Y, Orikasa S. Expression of cell cycle inhibitor p27 and Ki-67 in human adrenocortical neoplasms. Mod Pathol 1998;11(12):1165-70.

17. Stojadinovic A, Hoos A, Nissan A, Dudas ME, Cordon-Cardo $\mathrm{C}$, Shaha AR, et al. Parathyroid neoplasms: clinical, histopathological, and tissue microarray-based molecular analysis. Hum Pathol 2002;34:54-64.

18. Hoos A, Stojadinovic A, Singh B, Dudas ME, Leung DH, Ghossein RA. Clinical significance of molecular expression profiles of Hürthle cell tumors of the thyroid gland analyzed via tissue microarrays. Am J Pathol 2002;160:175-83.

19. Hoos A, Urist M, Stojadinovic A, Mastorides S, Dudas ME, Leung DHY, et al. Validation of tissue microarrays for immunohistochemical profiling of cancer specimens. Am J Pathol 2001;158:1245-51.

20. Schulik RD, Brennan MF. Long-term survival after complete resection and repeat resection in patients with adrenocortical carcinoma. Annu Surg Oncol 1999;6:719-26.

21. Harrison LE, Gaudin PB, Brennan MF. Pathologic features of prognostic significance for adrenocortical carcinoma after curative resection. Arch Surg 1999;134:181-5.

22. Kononen J, Bubendorf L, Kallioniemi A, Barlund M, Schraml $\mathrm{P}$, Leighton S, et al. Tissue microarrays for high-throughput molecular profiling of tumor specimens. Nat Med 1998;4: 844-7.

23. Kendrick ML, Lloyd R, Erickson L, Farley DR, Grant CS, Thompson GB, et al. Adrenocortical carcinoma: surgical progress or status quo? Arch Surg 2001;136:543-9.

24. Hoos A, Cordon-Cardo C. Tissue microarray profiling of cancer specimens and cell lines: opportunities and limitations. Lab Invest 2001;81:1331-8.

25. Reincke M. Mutations in adrenocortical tumors. Horm Metab Res 1998;30:447-55.

26. Levine AJ, Momand J, Finlay CA. The p53 tumor suppressor gene. Nature (Lond) 1991;351:453-6.

27. Momand J, Zambetti GP, Olson DC, Olson DC, George D, Levine AJ. The mdm-2 oncogene product forms a complex with the p53 protein and inhibits p53-mediated transactivation. Cell 1992;69:1237-45.

28. Zirbes TK, Baldus SE, Moenig SP, Nolden S, Kunze D, Shafizadeh ST, et al. Prognostic impact of p21/waf1/cip1 in colorectal cancer. Int J Cancer 2000;89:14-8.

29. Stein JP, Ginsberg DA, Grossfeld GD, Chatterjee SJ, Esrig D, Dickinson MG, et al. Effect of p21WAF1/CIP1 expression on tumor progression in bladder cancer. J Natl Cancer Inst 1998;90:1072-9.

30. Ogawa M, Maeda K, Onoda N, Chung YS, Sowa M. Loss of p21WAF1/DIP1 expression correlates with disease progression in gastric carcinoma. Br J Cancer 1997;75:1617-20.

31. Kawamata N, Morosetti R, Miller CW, Park D, Spirin KS, Nakamaki $\mathrm{T}$, et al. Molecular analysis of the cyclindependent kinase inhibitor gene p2 $7^{\text {Kip1 }}$, in human malignancies. Cancer Res 1995;55:2266-9.

32. Naruse I, Hoshino H, Dobashi K, Minato K, Saito R, Mori M. Overexpression of $\mathrm{p} 27^{\mathrm{Kip} 1}$ induces growth arrest and apoptosis mediated by changes of $\mathrm{pRb}$ expression in lung cancer cell lines. Int J Cancer 2000;88:377-83. 